\title{
Solution-Processed $\mathrm{VO}_{2} @ A g$ Nanoparticles for Modifying the Color of $\mathrm{VO}_{2}$ Smart Films
}

\author{
Xuan-Ming LU ${ }^{1,2, a}$, Xiu-Di XIAO ${ }^{1, b,{ }^{*},}$ Zi-Yi CAO ${ }^{1,3, c}$, Yong-Jun ZHAN ${ }^{1, d}$, \\ Hao-Liang $\mathrm{CHENG}^{1, \mathrm{e}}$, Gang $X \mathrm{U}^{1, \mathrm{f}}$
}

${ }^{1}$ Key Laboratory of Renewable Energy, Chinese Academy of Sciences; Guangdong Key Laboratory of New and Renewable Energy Research and Development; Guangzhou Institute of Energy

Conversion, Chinese Academy of Sciences, Guangzhou 510640, PR China.

${ }^{2}$ University of Chinese Academy of Sciences, Beijing 100049, China.

${ }^{3}$ Nano Science and Technology Institute, University of Science \& Technology of China, Suzhou 215123, China.

aluxuanming@foxmail.com, bxiaoxd@ms.giec.ac.cn, c237809861@qq.com, dzyj04dz@163.com, echenghl@ms.giec.ac.cn, ${ }^{\mathrm{f} x u g a n g @ m s . g i e c . a c . c n}$

*Corresponding author. E-mail address: xiaoxd@ms.giec.ac.cn

Keywords: Vanadium dioxide, Surface plasmon resonance, Spectrum modulation

Abstract. Recently, researchers focus on how to prepare practical $\mathrm{VO}_{2}$ films with varied colors. Herein, we developed a facile solution process to modify the spectrum of $\mathrm{VO}_{2}$ so that the color of $\mathrm{VO}_{2}$ film could be tuned. The $\mathrm{VO}_{2}$ nanoparticles were functionalized by the 3-aminopropyltrimethoxysilane(APTMS), and the reaction condition of APTMS functionalization was investigated. The $\mathrm{VO}_{2} @ A g$ was obtained by a two-step method. The UV-vis spectrum of $\mathrm{VO}_{2} @$ Ag exhibited an absorption band at $451 \mathrm{~nm}$, which meant the visible spectrum of $\mathrm{VO}_{2}$ could be modified by the Ag coating. Therefore, the $\mathrm{VO}_{2} @ A g$ films can display a corresponding complementary color. This technique will play an important role in practical application of $\mathrm{VO}_{2}$ smart films.

\section{Introduction}

Vanadium dioxide $\left(\mathrm{VO}_{2}\right)$ has been demonstrated to be a promising candidate material for the thermochromic smart films due to their reversible phase transition [1]. It can be transformed from monoclinic type $\mathrm{VO}_{2}(\mathrm{M})$ (space group: $\mathrm{P} 2_{1} / \mathrm{c}$ ) to tetragonal rutile type $\mathrm{VO}_{2}(\mathrm{R})$ (space group: $\left.\mathrm{P} 4_{2} / \mathrm{mnm}\right)$ at a critical temperature $\left(68^{\circ} \mathrm{C}\right.$ for bulk $\left.\mathrm{VO}_{2}\right)$, which results in an abrupt change in optical properties from transparent to translucent at the infrared spectral region. However, the $\mathrm{VO}_{2}$-based smart films have not been popular because of the high phase transition temperature, low visible transmittance, poor solar energy modulation ability, and undesirable color. The color of intrinsic $\mathrm{VO}_{2}$ film is not pleasant because it is brown. In a real application, we can find that the color of windows most used in the modern building is the blue color or other similar, which is more likely accepted by people. In this work, we focus on the color modulation of the $\mathrm{VO}_{2}$-based films.

Surface plasmon resonance (SPR) is a signature optical property of noble metal nanoparticles such as $\mathrm{Au}, \mathrm{Ag}$ and $\mathrm{Cu}$. The wavelength at absorption maximum $\left(\lambda_{\mathrm{SPR}}\right)$ and the absorption intensity are sensitive to many factors, including not only the properties of the metal particle but also the surrounding dielectric environment. $\mathrm{Xu}$ et al. utilized radio frequency magnetron sputtering to deposit $\mathrm{Ag}$ [2]or $\mathrm{Au}$ [3] nanoparticles on the $\mathrm{VO}_{2}$ films surface. Binionset al. prepared $\mathrm{VO}_{2} / \mathrm{Au}$ composite films with different color via chemical vapor deposition (CVD) [4, 5].The absorption band of the $\mathrm{VO}_{2}$ film had been changed with the sizes of metal nanoparticles varied. Thus, the film with different colors. However, whether magnetron sputtering or CVD needs harsh conditions, which is not conducive to practical production of large-scale and cost-effective smart films.

So far until now, there is few report involved in synthesis $\mathrm{VO}_{2} @$ metal nanoparticles via solution process. In this work, we proposed a solution processing method to modify the spectrum of the $\mathrm{VO}_{2}$, 
by coating $\mathrm{VO}_{2}$ with Ag nanoparticles. The $\mathrm{VO}_{2} @ A g$ exhibited an absorption peak at $451 \mathrm{~nm}$. It demonstrated that this method could modify the spectrum of the $\mathrm{VO}_{2}$ and tune the color of the $\mathrm{VO}_{2}$ films. This technique will play a role in practical application of vanadium dioxide smart windows.

\section{Experiment}

$0.2 \mathrm{M} \mathrm{NH}_{4} \mathrm{HCO}_{3}$ aqueous solution was dropped into $0.1 \mathrm{M} \mathrm{VOSO}_{4}$ aqueous solution. The greywhite precipitation was recovered by filtration and washed with copious amounts of water. For attaining $\mathrm{VO}_{2}(\mathrm{M})$, the greywhite precursor was redispersed in water and then transferred to a $100-\mathrm{mL}$ autoclave. The autoclave was maintained at $280{ }^{\circ} \mathrm{Cfor} 24 \mathrm{~h}$ and then cooled to room temperature naturally. Dark blue black precipitates were obtained and washed with deionized water and ethanol several times, and dried at $60^{\circ} \mathrm{C}$ for $8 \mathrm{~h} .0 .166 \mathrm{~g} \mathrm{VO}_{2}(\mathrm{M})$ above mentioned was dispersed in $200 \mathrm{~mL}$ absolute ethanol by ultrasonic for $30 \mathrm{~min}$.

Then, excess of 3-aminopropyltrimethoxysilane (APTMS) was added to $\mathrm{VO}_{2}$ nanoparticlesaqueous solution under vigorously stirred and the mixture was allowed to react for $12 \mathrm{~h}$ at the room temperature. The solution was gently refluxed for 1 additional hour in $80^{\circ} \mathrm{C}$ to enhance covalent bonding between the APTMS and the $\mathrm{VO}_{2}$ nanoparticles. The APTMS-functionalized $\mathrm{VO}_{2}$ nanoparticles were washed by ethanol at least 5 times to remove the unattached APTMS.

The ultrafine Ag nanoparticles as Ag seeds was produced by reduction of silver nitrate.Tannin acid was used as reductant. $0.01 \mathrm{~g}$ functionalized $\mathrm{VO}_{2}$ was added into $100 \mathrm{ml}$ deionized water and dispersed by ultrasonic for $30 \mathrm{~min}$. After adjusting the $\mathrm{pH}$, the solution was transferred into a flaskcontained Ag seeds, then stirred for $1 \mathrm{~h}$, and allowed standing for another $2 \mathrm{~h}$. The resulting was washed by water for several times until there was no free Ag nanoparticle.

Finally, the $\mathrm{VO}_{2} @ \mathrm{Ag}$ seeds particles were dispersed in $\mathrm{AgNO}_{3}$ aqueous solution, then the sodium borohydride as reductant was added into for growth of Ag seed and preparation of $\mathrm{VO}_{2} @ \mathrm{Ag}$ nanoparticles.

\section{Results}

\section{Reaction Condition of the Functionalization with APTMS.}

The functionalization was carried out by mixing the solution of nanoparticles with a certain amount ofAPTMS (sufficient to provide five monolayer coatings of the silica nanoparticles). The area on the nanoparticles surface covered by each APTMS molecule was assumed to be nominally 0.6 $\mathrm{nm}^{2}[6]$. Fourier transform infrared (FT-IR) spectra was used to detect the organic functional groups. Fig. 1 (black line) shows FT-IR spectra of intrinsic APTMS. The stretch mode of $-\mathrm{NH}_{2}$ and N-H groups of APTMS are observed at approximately $3356 \mathrm{~cm}^{-1}$ and $3284 \mathrm{~cm}^{-1}$, respectively. And the bands at $1595 \mathrm{~cm}^{-1}$ and $1471 \mathrm{~cm}^{-1}$ are due to the symmetric and asymmetric deformation mode of the hydrogen bonded amine group, respectively. However, there is no any band in in the spectrum (Fig.1 blue line) of Sample I (Table 1) near $3356 \mathrm{~cm}^{-1}$ or $3284 \mathrm{~cm}^{-1}$. It indicates that the functionalization was failed. 


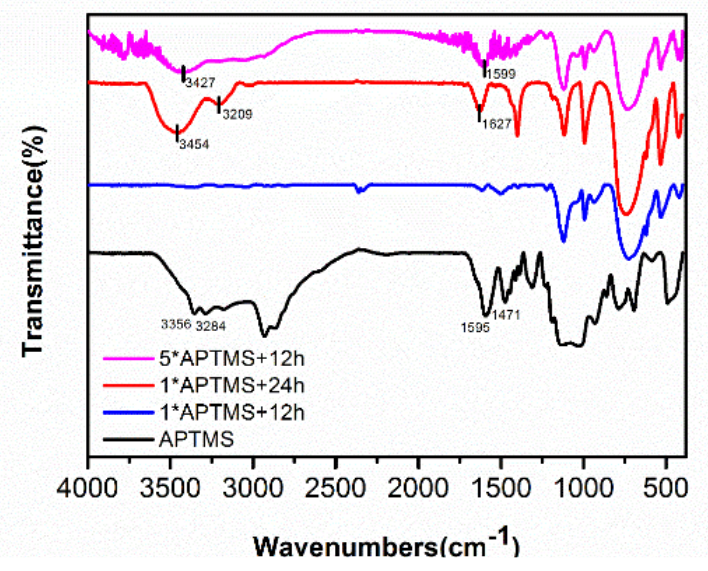

Fig. 1 FT-IR spectra of $\mathrm{VO}_{2}$ nanoparticles functionalized by APTMS with different concentration and time.

There may be two reasons for the failure of functionalization. First, the concentration of APTMS was too low. In the initial case, the addition amount of APTMS was the theoretical value above mentioned. In fact, considering the hydrolysis of APTMS and the surface activity of $\mathrm{VO}_{2}$, these factors make the amount of APTMS less than the amount actuallyrequired. Second, the reaction time is inadequate. It may need more energy or more time to bond. Thus, the reaction condition had been investigated. (Table 1)

Table 1. The reaction condition of APTMS functionalization.

\begin{tabular}{|l|l|l|l|c|}
\hline Sample & Amount of APTMS & Stirring time & Refluxed time & Functionalization \\
\hline I & 1 aliquot & $12 \mathrm{~h}$ & $1 \mathrm{~h}$ & $\times$ \\
\hline II & 5 aliquot & $12 \mathrm{~h}$ & $1 \mathrm{~h}$ & $\sqrt{ }$ \\
\hline III & 1 aliquot & $24 \mathrm{~h}$ & $1 \mathrm{~h}$ & $\sqrt{ }$ \\
\hline
\end{tabular}

The pink line and red line in Fig. 1are the FT-IR spectra of the results of increasing amount (Sample II) and extending time (Sample III), respectively. There are obviously difference from Sample I. The bands at $3427 \mathrm{~cm}^{-1}, 1599 \mathrm{~cm}^{-1}$ (pink line) and $3454 \mathrm{~cm}^{-1}, 1627 \mathrm{~cm}^{-1}$ (red line) prove that the APTMS was bonding on the $\mathrm{VO}_{2}$ surface. The band of $-\mathrm{NH}_{2}$ have shifted to higher wavenumbers from $3356 \mathrm{~cm}^{-1}$ to $3454 \mathrm{~cm}^{-1}$ (red line) and $3427 \mathrm{~cm}^{-1}$ (pink line). This shift is probably result from the existence of the $\mathrm{V}-\mathrm{O}$ bond. These results indicated that both the increasing the amount of APTMS and extending the reaction time could functionalized $\mathrm{VO}_{2}$ with APTMS successfully.

\section{The UV-vis spectra of $\mathrm{VO}_{2} @ A g$ seeds and VO $\mathrm{VO}_{2} @ A g$.}

UV-vis spectra is an efficient and precise method of characterization of noble metal nanoparticles because of the SPR. Based on Mie theory, the SPR frequency $\left(\lambda_{S P R}\right)$ is associated with the size, shape, dielectric constant of metal nanoparticle, and the dielectric constant of material around metal particles[7]. In order to prove the Ag seeds had already deposited on the $\mathrm{VO}_{2}$ surface, the UV-vis spectra of functionalized $\mathrm{VO}_{2}$, silver colloids and $\mathrm{VO}_{2} @ \mathrm{Ag}$ seeds at different temperature was measured, respectively (Fig.2). It can be found that there is no absorption peak in the curve of $\mathrm{VO}_{2}$, whether low or high temperature. And the $\lambda_{\mathrm{SPR}}$ of $\mathrm{Ag}$ seeds $\left(\lambda_{\mathrm{SPR}}=407 \mathrm{~nm}\right)$ at low temperature is consistent with it at high temperature. Because the test process was completed within 5 minutes, the coagulation 


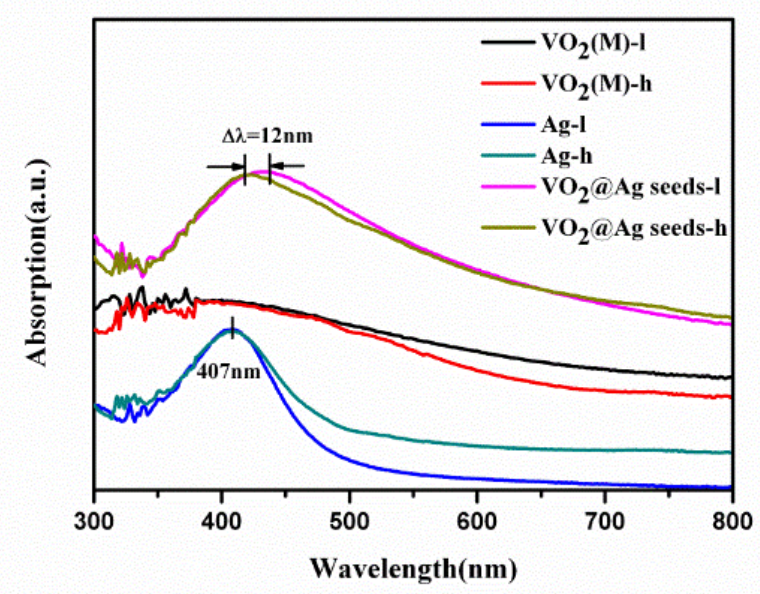

Fig. 2 Absorption spectra of Ag, $\mathrm{VO}_{2}, \mathrm{VO}_{2} @ \mathrm{Ag}$ seeds particles at low and high temperature.

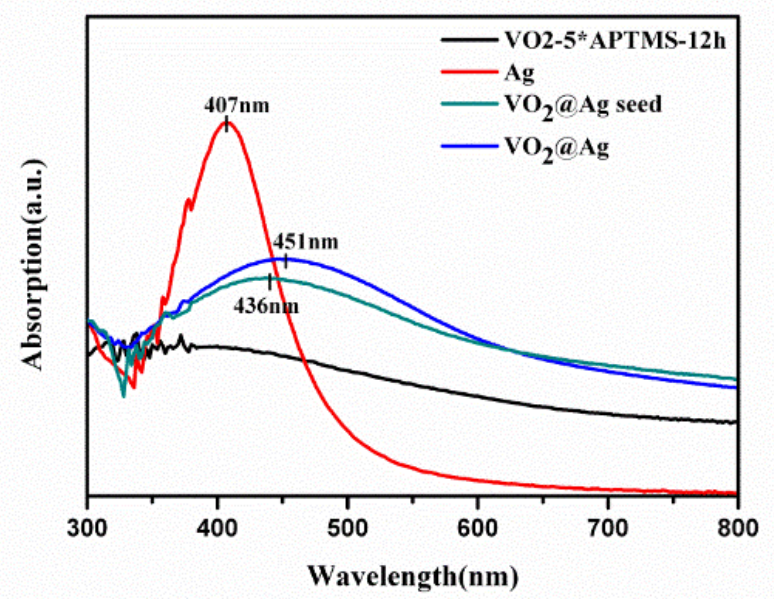

Fig. 3 Absorption spectra of Ag, $\mathrm{VO}_{2}, \mathrm{VO}_{2} @ A g$ seeds, and $\mathrm{VO}_{2} @ A g$ particles.

cause by heating could be ignored. However, the $\lambda_{\mathrm{SPR}}$ of $\mathrm{VO}_{2} @ \mathrm{Ag}$ seeds at low temperature is 436 $\mathrm{nm}$, and $424 \mathrm{~nm}$ at high temperature. Because the Fermi level of $\mathrm{VO}_{2}$ is lower than the Fermi level of Ag, the free electrons on the Ag nanoparticle surfaces will remove to the $\mathrm{VO}_{2}$ surfaces [8]. That results in decrease of electron density on the Ag nanoparticle surfaces. According the Mie theory, the $\lambda_{\text {SPR }}$ is inversely proportional to the square root of electron density. Therefore, the $\lambda_{\text {SPR }}$ shifted from $407 \mathrm{~nm}$ to $436 \mathrm{~nm}$ when the Ag particles attached the $\mathrm{VO}_{2}$. Additionally, the dielectric of $\mathrm{VO}_{2}$ (M) (low-temperature phase) is lower than $\mathrm{VO}_{2}(\mathrm{R})$ (high-temperature phase). On the basis of Mie theory mentioned above, the $\lambda_{\mathrm{SPR}}$ has a blue shift $\left(\Delta \lambda_{\mathrm{SPR}}=12 \mathrm{~nm}\right)$ due to the change of the dielectric constantof $\mathrm{VO}_{2}$. All the phenomena can be ascribed to that Ag nanoparticles deposited on the $\mathrm{VO}_{2}$ surfaces.

After the growth of $\mathrm{Ag}$ nanoparticles on $\mathrm{VO}_{2}$ surface, the $\lambda_{\text {SPR }}$ was shifted from $436 \mathrm{~nm}$ to 451 nm due to the size of Ag particles increase(Fig. 3). It implied that the $\lambda_{\mathrm{SPR}}$ of the $\mathrm{VO}_{2} @ \mathrm{Ag}$ could be easily controlled by the size of Ag particles, which was according to the centration of $\mathrm{AgNO}_{3}$. Therefore, the $\mathrm{VO}_{2}$-based smart films with varied colors could be prepared by our method.

\section{Conclusion}

In this work, we developed a facile method to tailor the undesired color of $\mathrm{VO}_{2}$-based smart film. $\mathrm{VO}_{2} @ A g$ core-shell nanoparticles were synthesized via solution process. The UV-vis spectrumof $\mathrm{VO}_{2} @ \mathrm{Ag}$ shows that there is an absorptionband at $451 \mathrm{~nm}$, which means the visible spectrum of 
$\mathrm{VO}_{2}$ could be modified by the Ag coating. Therefore, the $\mathrm{VO}_{2} @ A g$ films may display a corresponding complementary color. This work paves the way to the scale-up and fast production of $\mathrm{VO}_{2}$-based smart film with varied colors.

\section{Acknowledgement}

This work was supported by the Major Science and Technology Projects of Guangdong Province (No. 2013A011401011), Science and Technology Project of Guangdong Province (No. 2014A010106018), Pearl River Star of Science and Technology (No. 2014J2200078), CAS Key Laboratory of Renewable Energy Fund of GIEC (No. y507j41001),National Natural Science Foundation of China (No. 51506205), Guangdong Joint Innovation Project in Guangdong Province (No. 2014B050505015), Construction Project of Guangdong Solar Thermal and Solar Thermal Advanced Materials Engineering Technology Research Center (No. 2014B090904071).

\section{References}

[1] F.j. Morin, Oxides which show a metal-to-insulator transition at the Neel temperature, Phys. Rev. Lett. 3 (1959) 34-36.

[2]G. Xu, C.M. Huang, P. Jin, M. Tazawa, D.M. Chen, Nano-Ag on vanadium dioxide. I. localized spectrum tailoring, J. Appl. Phys. 104 (2008) 053101-053101-6

[3] G. Xu, C.M. Huang, M. Tazawa, P. Jin, L.H. Chen, Tunable optical properties of nano-Au on vanadium dioxide, Opt. Commun. 282 (2009) 896-902.

[4] R. Binions, C. Piccirillo, R.G. Palgrave, I.P. Parkin,Hybrid aerosol assisted and atmospheric pressure CVD of gold-doped vanadium dioxide, Chem. Vapor Depos. 14 (2008) 33-39.

[5] M. Saeli, C. Piccirillo, I.P. Parkin, I. Ridley, R. Binions, Nano-composite thermochromic thin films and their application in energy-efficient glazing, Sol. Energ. Mat. Sol. C. 94 (2010) 141-151.

[6] T.G. Waddell, D.E. Leyden, M.T. DeBello, The nature of organosilane to silica-surface bonding, J. Am. Chem. Soc. 103 (1981) 5303-5307.

[7] G. Mie, Beiträge zur optik trüber medien, speziell kolloidaler metallösungen, Ann. der Phys. 330 (1908) 377-445.

[8] G. Xu, C.M. Huang, M. Tazawa, P. Jin, D. M. Chen, L. Miao, Electron Injection Assisted Phase Transition in a Nano-Au- $\mathrm{VO}_{2}$ Junction, Appl. Phys. Lett. 93 (2008) 1911. 